Bundesgesundheitsbl -

Gesundheitsforsch - Gesundheitsschutz

2000 • 43:519-524 @ Springer-Verlag 1999

In der Diskussion

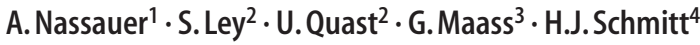

${ }^{1}$ Robert Koch Institut

${ }^{2}$ Deutsches Grünes Kreuz

${ }^{3}$ Deutsche Vereinigung zur Bekämpfung der Viruskrankheiten e.V.

${ }^{4}$ Universitäts-Kinderklinik, Kiel

\title{
Mehr Rechtssicherheit beim Impfen?
}

\section{Ein Diskussionsbeitrag}

erfahren vor den ordentlichen Gerichten mit hohen Schmerzensgeldforderungen gegen Ärzte durch Patienten, die einen Impfschaden geltend machten, haben stets große Beachtung gefunden. In Erinnerung sind z.B. die Klage eines Jugendlichen, der im zeitlichen Zusammenhang mit einer Mumps-Schutzimpfung an einem Diabetes mellitus erkrankte (die Entscheidungen des LG Gießen und des OLG Frankfurt sind nicht veröffentlicht) und die eines nicht gegen Poliomyelitis geimpften Erwachsenen, der mehrfach Kontakt zu einem frisch mit OPV geimpften Säugling hatte und eine schwere Kontaktpoliomyelitis erlitt [1]. Beim Diabetes mellitus wurde die Klage in zweiter Instanz abgewiesen. In dem berühmten Fall zur Kontaktpoliomyelitis hat der $\mathrm{BGH}$ wegen unzureichender Aufklärung dem Geschädigten Schmerzensgeld zugesprochen.

Auf Fortbildungsveranstaltungen wird immer wieder berichtet, dass zurzeit mehrere Haftpflichtprozesse gegen Ärzte wegen schwerer Gesundheitsschäden durch Schutzimpfungen anhängig sind. Besonders Kinderärzte sind verunsichert und befürchten Entscheidungen, die sie finanziell ruinieren würden [2]. Der Präsident des Berufsverbandes der Kinder- und Jugendärzte wird mit den Worten zitiert, dass sich derzeit mehrere tausend Ärzte vor Gericht verantworten müssten, u. a. weil sie angeblich ihre Aufklärungspflicht beim Impfen vernachlässigt hätten oder ihr gar nicht nachgekommen seien [3]. Richtig ist, dass genaue Zahlen der Klagen gegen Ärzte wegen Behandlungsfehlern nicht bekannt sind. 1997 lautete die Schätzung 10 ooo bis 15000 pro Jahr [4].

Für 1998 wird mitgeteilt, dass jährlich bei deutschen Haftpflichtversicherern ca. 10 ooo Schadensmeldungen aus der gesamten Medizin eingingen. Allerdings kam es nur in rund 10\% der Fälle zu einem Gerichtsverfahren [5]. Im Dezember 1999 titelte "Die Welt": "Jährlich 30 ooo Klagen wegen ärztlicher Kunstfehler. Jeder dritte Fall wird von den Gerichten in Deutschland im Sinne der Patienten entschieden" [6]. Die Zahlen variieren nicht unerheblich. Zu Beginn des Jahres beschäftigte sich "Der Spiegel" mit Kunstfehlervorwürfen. In einer Grafik wird dargestellt, dass sich ca. zwei Drittel aller Forderungen an Chirurgen richteten, Pädiater waren lediglich in $1,4 \%$ aller Fälle betroffen. Unter Annahme der ungünstigsten hier aufgeführten Zahlen würden also 420 Kinderärzte pro Jahr mit Forderungen wegen fehlerhafter Behandlung konfrontiert (diese betreffen nicht nur Schutzimpfungen, sondern die gesamte Pädatrie!), nur ein Bruchteil davon endet allerdings vor Gericht.

\section{"Jährlich gehen bei deutschen Haftpflichtversicherern \\ ca. 10000 Schadensmeldungen aus der gesamten Medizin ein. Nur in ca. 10\% der Fälle kam es jedoch zu einem Gerichtsverfahren!"}

So ist die Angst, Tausende von Ärzten müssten sich vor Gericht verantworten - auch unter der Annahme, dass die Prozessfreudigkeit und der Verfahrensstau bei den Gerichten zunehmen nicht begründet.

\section{Persönliche Haftung bei Impfschäden}

1998 schrieben Berliner Kinderärzte im Rahmen einer Unterschriftensammlung an das Bundesgesundheitsministerium: "Wir fordern eine eindeutige Gesetzeslage, die besagt, dass bei Impfschäden, d.h. bei spezifisch impfstoffbedingten Nebenwirkungen und Folgeerscheinungen, der Impfarzt nicht persönlich haftbar gemacht werden darf. Es haftet ausschließlich der Staat. Die Aufklärungspflicht obliegt dem Staat" [9]. Diese Forderung geht prinzipiell zutreffend von der Annahme aus, dass Schutzimpfungen auch eine öffentliche Angelegenheit sind [10]. Gemäß $\$ \$ 51 / 52$ BSeuch G (bzw. künftig $₫ 60$ JfSG (Infektionsschutzgesetz), sobald dieser Gesetzentwurf im Deutschen Bundestag verabschiedet ist) entschädigt der Staat einen eventuellen Gesundheitsschaden im Rahmen eines gesetzlichen Aufopferungsanspruchs.
Dr. Alfred Nassauer

Robert Koch-Institut,

Abteilung für Infektionskrankheiten,

Stresemannstraße 90-102, 10963 Berlin 
Eine ausschließliche Haftung des Staates verkürzte jedoch das verfassungsrechtlich garantierte Rechtsschutzinteresse der Patienten und das Prinzip der Gewaltenteilung (Art. 20 Abs. 2 und 3, Art. 101 und Art. 103 GG). Insofern kann die Forderung nach ausschließlicher Haftung des Staates nicht Bestand haben. Auch wird verkannt, dass zivilrechtliche Verfahren wegen deliktischer Haftung oder Strafprozesse nur dann zum Nachteil von Ärzten entschieden werden, wenn ihnen schuldhaftes Handeln (Vorsatz oder Fahrlässigkeit) nachgewiesen werden kann. Gelingt dieser Nachweis nicht, bleibt es zwar bei der erheblichen psychischen Belastung, die jedes Verfahren mit sich bringt; Schadenersatz oder Schmerzensgeld sind nicht zu leisten, auch eine Strafe kann nicht verhängt werden.

Dass die Aufklärungspflicht dem Staat obliege, wird durch den neuen Rechtsgedanken in $\$ 20$ Abs. 1 E - JfSG (Entwurf des IfSG) gestützt, wonach der öffentliche Gesundheitsdienst die Bevölkerung über Schutzimpfungen informiert. Diese Vorschrift ersetzt jedoch nicht den Anspruch eines jeden Patienten, vor einer medizinischen Maßnahme individuell seinen Bedürfnissen entsprechend aufgeklärt zu werden $(\$ 1 \mathrm{a}$ Musterberufsordnung der Deutschen Ärzteschaft).

\section{Sind Impfungen eine Körperverletzung?}

Dass durch ärztliche Maßnahmen seit einem Urteil des Reichsgerichts im Jahr 1894 der Tatbestand der Körperverletzung erfüllt ist, muss für Ärzte irritierend sein. Lösungsvorschläge wurden durch die Rechtswissenschaft unterbreitet, und auch das Justizministerium hat 1996 einen Vorstoß unternommen, nur die "eigenmächtige Heilbehandlung" und die "fehlerhafte Heilbehandlung" als spezielle Tatbestände in das Strafgesetzbuch aufzunehmen. Nach Kritik von Ärzten wie Juristen wurde der Entwurf allerdings wieder ad acta gelegt, so dass die Rechtslage bzgl. des Tatbestandes “Körperverletzung” weiter besteht [11]. Auch eine Schutzimpfung erfüllt den äußeren Tatbestand der Körperverletzung, sie ist jedoch nicht rechtswidrig, wenn ein Rechtfertigungsgrund vorliegt. Bei ärztlichen Eingriffen wird dieser regelmäßig durch die Einwilligung des Pati- enten geschaffen [12].

Wenn also der Berufsverband der Kinder- und Jugendärzte in der Jahrespresseerklärung 1999 fordert, Impfungen dürften keine Körperverletzung sein, greift er einen alten Streit auf [13], der im Ergebnis zwar nicht entschieden ist, aber im Kern geht es um eine rechtsdogmatische Frage, die in der Praxis keine entscheidende Bedeutung haben sollte.

\section{Studium von Urteilen}

Einen Artikel aus der Molekularbiologie oder Immunologie zu verstehen oder nachzuvollziehen, fällt hin und wieder schwer. Niemand käme darauf, in einem Rechtsstreit (sofern es dabei auf seine Aussagen ankommt) eine solche Publikation dem Gericht oder den Anwälten mit der Bemerkung zu überreichen, dort sei alles wissenschaftlich erklärt, und die Verfahrensbeteiligten könnten sich danach selbst informieren. Natürlich wird erwartet, dass sachkundige Gutachter Richtern und Parteien die Sachlage verdeutlichen. Oft genug geht es Ärzten bei der Lektüre von Urteilen ähnlich. Die Rechtswissenschaft hat ihre eigene Terminologie und Sprache entwickelt, die Laien befremdet bzw. für sie unverständlich erscheint. Allerdings darf nicht außer Acht gelassen werden, dass in Gerichtsurteilen - auch im Medizinrecht den streitenden Parteien (von denen regelmäßig nur eine aus der Medizin kommt) Entscheidung, Sachverhalt und Begründung laienverständlich zu erläutern ist. Dieser Weg (Verdeutlichung von Aussagen für Nichtfachleute) ist in medizinischen Fachpublikationen meist nicht zu beschreiten.

\section{“Verkürzte Medien-Meldungen führen leicht zu Fehl- interpretationen, Ängsten und Vorurteilen, die sich durch das Studium des Originalurteils beseitigen lassen."}

Über Haftpflichtprozesse gegen Ärzte wird in der Tagespresse und Medizin dann berichtet, wenn Medizinern schuldhaftes Handeln nachzuweisen war [14]. Unterliegt der klagende Patient, ist dies in der Regel keine Notiz wert. So entsteht zwangsläufig der Eindruck, Ärzte verlören meist die Prozesse. Dem kann nur entgegengetreten werden, indem einschlägige Urteile in medizinischen Fachzeitschriften besprochen und erläutert werden $[15,16]$. In diesen beiden Fundstellen wird durch R. Günter der Sachverhalt unterschiedlicher Entscheidungen zu DPT-Schutzimpfungen berichtet, in längeren Passagen aus den Urteilen zitiert, und durch Fettdruck wird der juristische Laie darauf hingewiesen, worauf es ankommt [17].

\section{Juristische Datenbanken}

Die Recherche in Internetdatenbanken ist schon wegen des Zugangs nicht immer ohne Hürden, aber mit fachlicher Hilfe dennoch möglich. Für die Jahre 1997 bis 1999 wurden unter den Stichworten Impfung, Schutzimpfung und Impfschäden 47 Urteile gefunden. Davon waren 30 Entscheidungen aus der Sozialgerichtsbarkeit und betrafen fast ausschließlich Fragen des Kausalitätsnachweises, inwieweit eine Schutzimpfung die Ursache für einen Impfschaden im Rahmen des öffentlich-rechtlichen Aufopferungsanspruchs (\$\$ 51/52 BSeuchG) war. Acht Verfahren waren von Verwaltungsgerichten zu entscheiden; hier ging es wesentlich um Fragen aus dem Arzneimittelrecht, allein vier Entscheidungen betrafen Schutzimpfungen in der Veterinärmedizin.

Neun Entscheidungen waren aus der Zivilgerichtsbarkeit. Im Ausdruck der Datenbank fand sich kein Urteil aus dem Strafrecht.

Die neun Zivilgerichtsurteile behandelten im Einzelnen:

D zwei Forderungen aus Reiserücktrittskostenversicherungen bei (angeblicher) Impfunverträglichkeit,

D eine Autovakzinetherapie als notwendige Heilbehandlung bei AIDS,

D die Frage des Wettbewerbsverstoßes bei vergleichender Werbung für ein Arzneimittel,

D ein Verfahren aus Österreich, wobei es vor dem OLG Wien um eine kreditschädigende Aussage über ein Arzneimittel ging,

D eine Patentverletzung hinsichtlich des deutschen Teils eines europäischen Patents,

D die Rechtzeitigkeit der Impfung gegen die Aujeszkysche Krankheit bei Schweinen. 


\section{Schadensersatz- und Schmerzens- geldforderungen}

Nur in zwei Fällen ging es um Schadensersatz- und Schmerzensgeldforderungen durch Patienten gegen Ärzte wegen einer behaupteten Impfschädigung. Beide Male waren die Kläger nicht erfolgreich:

D Das LG Waldhut-Tiengen hat die Klage, dass eine FSME-Schutzimpfung zu einer Enzephalomyeloradikulitis geführt habe, mit der Begründung abgewiesen, dass der Kläger den notwendigen Nachweis der Kausalität nicht erbracht habe und auch (im Rahmen der Beweiserleichterung) eine überwiegende Wahrscheinlichkeit der Impfung als Ursache für den Impfschaden nicht angenommen werden könne [18].

D In einem Verfahren vor dem OLG Stuttgart waren die Erben einer Kinderärztin verklagt, weil eine DPTSchutzimpfung Ursache für eine danach eingetretene statomotorische Retardierung gewesen sein soll. Auch dieses Gericht hat festgestellt: Die Ursächlichkeit der Impfung für die Schädigung eines Kindes hat (im Zivilprozess) der Kläger zu beweisen. Dies gilt sowohl für den Vorwurf ungenügender oder fehlender Aufklärung als auch für den Behandlungsfehlervorwurf. Eine akute Impfenzephalopathie geht mit bestimmten Symptomen einher. Fehlen derartige Symptome, kann nicht von der Ursächlichkeit der Impfung für die Schädigung des Kindes ausgegangen werden (Leitsätze des Bearbeiters) [19].

Selbstverständlich ist diese Sichtung von Urteilen aus einer Datenbank keine statistische Analyse, die eine prozentuale Aussage zuließe, wie oft Ärzte wegen fehlerhafter Impfung und Aufklärung Leistungen an Patienten erbringen müssen. Mit der Darstellung soll vielmehr verdeutlicht werden, dass nur die eingehende Betrachtung von Urteilstexten durch Ärzte selbst oder nach Aufbereitung durch juristisch kundige Autoren davor bewahrt, Missverständnisse zu schüren oder Behauptungen aufrecht zu erhalten, die einer Prüfung nicht stand halten.

\section{Die Rolle der Gutachter}

Die beiden zuletzt genannten Urteile aus Stuttgart und Waldshut setzen sich intensiv mit Aussagen der Gutachter auseinander, und zweifellos kam es für den Ausgang des Verfahrens entscheidend auf deren Sachverstand an. Mangelnde Sachkunde und Befangenheit von Gutachtern wird - zu Recht - beklagt [20].

Einen Ausweg weist zukünftig $\$ 20$ Abs. 2 E-IfSG, der die Ständige Impfkommission am Robert Koch-Institut (STIKO) beauftragt, "Kriterien zur Abgrenzung einer üblichen Impfreaktion und einer über das übliche Ausmaß einer Impfreaktion hinausgehenden gesundheitlichen Schädigung zu entwickeln", die zuverlässiger als bisher eine Bewertung ermöglichen sollen.

\section{Aufklärung vor Schutz- impfungen durch Merkblätter}

In den hier aufgeführten Urteilen $[1,18$, 19,10,12,17 mit den dortigen Nachweisen] kam es für den Ausgang des Verfahrens auf eine sachgerechte Aufklärung des Impfwilligen vor der Schutzimpfung an. Auch für die Beurteilung der Rechtswidrigkeit einer Schutzimpfung als Straftatbestand sind Einwilligung und Aufklärung ganz entscheidend.

\section{"Was Gegenstand der Aufklärung sein soll, muss formuliert werden."}

Deshalb sind Forderungen aus dem Kreis der Kinderärzte völlig berechtigt, dass deutlicher als bisher Kriterien zu formulieren sind, was Gegenstand der Aufklärung sein soll [13]. In dieser Presseerklärung wird eine gesetzliche Regelung gefordert. Ob dies der richtige Weg ist, erscheint zweifelhaft, da Gesetze in der Regel allgemein (für alle denkbaren Sachverhalte) gelten müssen und deshalb kaum in der Lage sind, Ärzten die gewünscht einfache und klare Richtschnur zu Art und Umfang der Aufklärung vor Schutzimpfungen an die Hand zu geben. Vielmehr wird die geforderte Sicherheit durch die Rechtsprechung erreicht, die verständlich formuliert und in Urteilen den Ausgleich der widerstreitenden Interessen beschreibt.

Am 20.4.2000 fand sich im Videotext der ARD (sinngemäß) die Mittei- lung, dass der Bundesgerichtshof entschieden habe, dass eine Aufklärung vor einer Routineimpfung durch ein Merkblatt ausreiche. Am 21.4. wurde durch die Entscheidungsversendungsstelle des BGH der vollständige Text zur Verfügung gestellt. Das Urteil ist ein Vorbild hinsichtlich Klarheit und Verständlichkeit für den juristischen Laien und ist in seinen Aussagen (als höchstrichterliche Entscheidung) eine zuverlässige Richtschnur für die ärztliche Aufklärung [21].

\section{Ein Beispiel}

Klägerin war ein Kind, das im Februar 1994 in der 34. Schwangerschaftswoche geboren wurde. Es erhielt im Mai 1994 eine erste Impfung gegen DPT-Hib und eine Dosis OPV-Impfstoff. Zuvor hatte die Mutter ein Merkblatt von der beklagten Kinderärztin erhalten, von dem sie im Wartezimmer Kenntnis nahm, ohne es $\mathrm{zu}$ unterschreiben. Beim Eintritt in das Behandlungszimmer war sie dann von der Kinderärztin befragt worden, ob sie das Merkblatt gelesen habe, was sie bejahte. Nach der Untersuchung der Klägerin hatte die Beklagte anschließend erklärt, wenn die Mutter es wolle, könne man jetzt impfen. Fünf Wochen später kam die Mutter mit dem Kind wegen eines Hautausschlages (leider ist dies im Text des Sachverhalts nicht näher erläutert) erneut in die Sprechstunde und erhielt bei dieser Gelegenheit eine zweite OPV-Dosis. Fünf Tage später trat Fieber auf, und später wurde eine Schonhaltung des linken Beines festgestellt. Bei den nachfolgenden Untersuchungen wurde eine Impfpoliomyelitis diagnostiziert, und das Versorgungsamt hat eine Schadensrente bewilligt (s. Fallbericht).

\section{Fallbericht Impfpoliomyelitis}

Aus ärztlicher Sicht ist der Fall folgendermaßen zu beurteilen: Das am 8.2.94 geborene Mädchen wurde am 11.5.94 also altersgerecht - zum ersten Mal mit OPV geimpft. Am 13.6. erfolgte die 2. OPV-Gabe. Am 18.6. trat Fieber auf, am 25.6., also 44 Tage nach der 1 . und 12 Tage nach der 2. Impfung, wurde eine Schonhaltung des linken Beines berichtet. Diese nahm in der Folge zu, so dass laut Akte eine Kinderlähmung mit einer Minderung der Erwerbsfähigkeit von $80 \%$ festgestellt wurde.

Eine typische Impfpoliomyelitis 
kann nur dann angenommen werden, wenn die Lähmungen innerhalb von sieben bis 30 Tagen p. v. auftreten. Die Komplikation muss also Folge der 2. OPV-Gabe sein. Dies ist recht selten, wenn auch nicht auszuschließen: eine Impfpoliomyelitis beim Geimpften wird bei 1:5 Mio. Impfungen beobachtet [23]. Die Rate nach der 1 . Impfung ist dabei mit 1:520 ooo deutlich höher als die von 1:12,3 Mio. bei folgenden Impfungen [24]. Neben der typischen Impfpoliomyelitis wird in dem Urteil noch die Frage eine Guillain-Barré-Syndroms (GBS)als Ursache der Schädigung aufgeworfen. Eine finnische Studie, die zunächst einen Kausalzusammenhang von demyelinisierenden Erkrankungen mit der OPV-Impfung vermuten ließ, wurde später von denselben Autoren widerlegt [25]. Aus heutiger Sicht hätte ein GBS also als Impfschaden abgelehnt werden müssen. An dieser Stelle sollte noch einmal darauf hingewiesen werden, dass bei der Anerkennung eines Impfschadens nur ein Kausalzusammenhang mit der Impfung wahrscheinlich gemacht werden muss, während es bei einer zivilrechtlichen oder strafrechtlichen Beurteilung gilt, ein schuldhaftes Handeln und den Zusammenhang mit der Impfung zu beweisen. Im letzteren Fall kommt der exakten Diagnose also eine wesentlich höhere Bedeutung zu.

Die Klägerin hat der Beklagten eine fehlerhafte Behandlung und unzureichende Aufklärung vorgeworfen, auch habe die notwendige Einwilligung des Vaters gefehlt. Die Schmerzensgeldforderung betrug DM 100 ooo,-; außerdem sollte festgestellt werden, dass die Beklagte für alle Folgeschäden aus den Polioimpfungen haftbar sei. Der BGH hat die Klage gegen das Berufungsurteil zurückgewiesen. Die Entscheidungsgründe enthalten zahlreiche wertvolle Hinweise auf die im Einzelnen eingegangen werden soll, auch wenn es sich um Feststellungen handelt, die teilweise bereits in früheren Entscheidungen veröffentlicht wurden.

\section{Ist die Zustimmung beider Elternteile zwingend?}

Zu dem Thema, ob vor der Impfung die Zustimmung beider Elternteile zwingend nötig sei, sagt das Gericht: “... man (wird) im Allgemeinen davon ausgehen können, dass der mit dem Kinde beim
Arzt erscheinende Elternteil ermächtigt ist, die Einwilligung in die ärztliche Behandlung für den abwesenden Elternteil mit zu erteilen, worauf der Arzt in Grenzen vertrauen darf, solange ihm keine entgegenstehenden Umstände bekannt sind (BGHZ 105, 45). Dies gilt jedenfalls in Routinefällen, zu denen auch die Routineimpfung gehört.”

\section{Routineimpfung}

Zitat: "Bei der durchgeführten Schluckimpfung gegen Kinderlähmung handelt es sich um eine Routineimpfung. Sie war von der STIKO seit langem empfohlen und wurde auch in Baden-Württemberg von der zuständigen Gesundheitheitsbehörde gem. $\$ 14$ Abs. 3 BSeuchG insbesondere 1994 öffentlich empfohlen. Sie wurde seit Einführung des oralen Polioimpfstoffes 1962 millionenfach durchgeführt”.

\section{Aufklärungsumfang}

Beim Thema Aufklärung betont das Gericht zunächst noch einmal, dass es hierbei nicht darauf ankommen kann, ob eine Komplikation nur die "äußerst seltene Folge" einer Impfung darstellt und fährt fort: "Entscheidend für die ärztliche Hinweispflicht ist nicht ein bestimmter Grad der Risikodichte, insbesondere nicht eine bestimmte Statistik. Maßgebend ist vielmehr, ob das betreffende Risiko dem Eingriff spezifisch anhaftet $^{1}$ und bei seiner Verwirklichung die Lebensführung des Patienten besonders belastet. Der Senat hält daher daran fest, dass grundsätzlich auch über derartige äußerst seltene Risiken aufzuklären ist. Das gilt entgegen der Auffassung der Revision und entsprechender Äußerungen im Schrifttum (Deutsch VersR

\footnotetext{
${ }^{1}$ Die folgenden Risiken waren für die orale Poliomyelitis-Impfung als spezifisch anzusehen (Quast,U. et al., Hippokrates-Verlag Stuttgart, 1997, 107 ff): Fieber, allgemeines Unwohlsein, Durchfälle, Impfpoliomyelitis. Im Jahre 1994 war evtl. noch das GBS zu erwägen. Beides wurde mit dem Begriff “Lähmungen" nach Meinung des BGH ausreichend im Merkblatt beschrieben. Nicht aufgeführt im fraglichen Merkblatt der Kinderärzte war allerdings eine weitere spezifische Komplikation: die Impfkontaktpoliomyelitis. Dies wurde in der Revision und in dem BGHUrteil zu Recht bemängelt, was allerdings für die hier zu treffende Entscheidung irrelevant war, weil dieser Schaden nicht eintrat.
}

1998, 1053, 1057) auch für öffentlich empfohlene Impfungen."

Diese Einschränkung ist von Bedeutung, da E. Deutsch in dem oben genannten Aufsatz für öffentlich empfohlene Impfungen eine Aufklärungspflicht über seltene, nicht aber auch über sehr seltene Nebenwirkungen (jede - auch relativ unverständliche - Eventualität) postuliert hat. Dieser Versuch einer Differenzierung, die gerade von Kinderärzten als Erleichterung empfunden wurde, ist damit nicht akzeptiert worden.

\section{Inhalt der Aufklärung}

\section{Risiken}

Zu Recht ist aus der Ärzteschaft immer wieder kritisiert worden, dass der ausführliche Hinweis auf Komplikationen dann Eltern davon abhalte, einer Impfung zuzustimmen. In diesem Zusammenhang ist folgende Passage aus dem Urteil wichtig: "Nach der Rechtsprechung des Senats braucht der Patient nur im Großen und Ganzen über Chancen und Risiken der Behandlung aufgeklärt zu werden. Nicht erforderlich ist die exakte medizinische Beschreibung der in Betracht kommenden Risiken (BGHZ90,103,106). Der Hinweis auf Lähmungen umfaßte daher auch solche aufgrund eines Guillain-Barré-Syndroms, so dass eine gesonderte Aufklärung hierüber nicht geboten war.” Das im konkreten Fall verwendete Merkblatt lautete: "Die Impfung wird normalerweise komplikationslos vertragen. Öfters werden als normale Reaktion breiige Stühle beobachtet, selten treten fieberhafte Reaktionen auf, extrem selten Lähmungen (ein Fall auf 5 Mio. Impfungen)."

Eine Aufklärung im Großen und Ganzen zwingt gerade nicht zur langatmigen Erläuterung verschiedener Krankheitsbilder und umgeht die Überbetonung der Risiken im Gespräch.

\section{Krankheitsbild}

Über die Krankheit Poliomyelitis wird in dem genannten Merkblatt gesagt: "Kinderlähmung ist eine Viruserkrankung, die zu bleibenden Lähmungen und sogar zum Tode führen kann. Eine ursächliche Behandlung gibt es nicht. Vor Einführung der Impfung gab es jährlich Tausende von Erkrankungen 
und hunderte von Todesfällen.” Das Gericht bescheinigt dem Merkblatt, dass es "das Risiko der Erkrankung an Kinderlähmung nicht ... in unzutreffender Weise dramatisiert habe."

\section{Impfempfehlungen der STIKO}

Im weiteren Verlauf der Begründung geht das Gericht auf die STIKO-Empfehlungen zur Polioschutzimpfung ein und vollzieht die epidemiologische Begründung nach, die 1998 zur Umstellung von OPV auf IPV geführt hat und fährt fort: "Überdies ist zu beachten, dass ... die Empfehlungen der STIKO medizinischer Standard sind. Da die STIKO ... 1994 die Impfung ab dem 3. Lebensmonat unter Anwendung des oralen Poliomyelitis-Impfstoffs empfahl und die zuständigen Gesundheitsbehörden der Länder dem folgend diese Impfmethode gem. $\$ 14$ Abs. 3 BSeuchG öffentlich empfahlen, war dies für den jeweiligen Kinderarzt maßgebend."

Damit sind die STIKO-Empfehlungen auch in der höchstrichterlichen Rechtsprechung als medizinischer Standard [22] ausdrücklich anerkannt. Dies hat nach Auffassung des Gerichts zur Folge, dass in dem Merkblatt die Behandlungsalternative mit IPV seinerzeit nicht ausdrücklich erwähnt werden musste.

\section{Rechtzeitigkeit der Aufklärung}

Da bei den Impfterminen nach dem Impfkalender der STIKO bis zu sechs Impfungen verabreicht werden, wurde immer wieder vorgeschlagen, schon bei der U3 Informationen den Eltern mitzugeben, damit sie genug Zeit für eine Entscheidung hätten. Auch dies ist nach dem neuen Urteil nicht erforderlich: "Nach gefestigter Rechtsprechung reicht bei ambulanten Eingriffen grundsätzlich eine Aufklärung am Tage des Eingriffs aus... . Es handelte sich um eine Routineimpfung, bei der den Eltern der Entscheidungskonflikt aufgrund der von den Gesundheitsbehörden vorgenommenen Impfempfehlung weitgehend abgenommen war." Und an anderer Stelle sagt das Gericht außerdem: "Die Notwendigkeit der Impfung war in der Bevölkerung seit langem allgemein anerkannt .... Bei dieser Sachlage konnte die Beklagte davon ausgehen, dass auch die Mutter der Klägerin mit der Impfung vertraut und über die allseits akzeptierte Notwendigkeit im Bilde war."

\section{Merkblatt und Gespräch?}

Zitat: "Nach der Rechtsprechung bedarf es zum Zwecke der Aufklärung des vertrauensvollen Gesprächs zwischen Arzt und Patienten. Das schließt jedoch keineswegs die Verwendung von Merkblättern aus, in denen die notwendigen Informationen zu dem Eingriff einschließlich seiner Risiken schriftlich festgehalten sind. Derartige schriftliche Hinweise sind heute weitgehend üblich und haben den Vorteil einer präzisen und umfassenden Beschreibung des Aufklärungsgegenstandes sowie der für den Arzt wesentlichen Beweisbarkeit. Sie sind insbesondere bei Routinebehandlungen, also auch bei öffentlich empfohlenen Impfungen, am Platze. Freilich vermögen solche Merkblätter nicht das erforderliche Arztgespräch zu ersetzen, doch gebietet dieses Erfordernis nicht in jedem Falle eine mündliche Erörterung der Risiken. Unter Umständen (wie bei anderen Routinemaßnahmen) kann der Arzt ausnahmsweise davon ausgehen, dass der Patient auf eine zusätzliche Risikodarstellung keinen Wert legt. Es kann genügen, wenn dem Patienten nach schriftlicher Aufklärung Gelegenheit zu weiteren Informationen durch ein Gespräch mit dem Arzt gegeben wird. Dies entspricht auch den Empfehlungen der STIKO [26].

\section{Mehrfache Aufklärung?}

Gerade in Fortbildungsveranstaltungen wird immer wieder gefragt, ob bei jeder Impfung im Rahmen einer Grundimmunisierung eine erneute Aufklärung erfolgen müsse. Auch hierzu hat sich das Gericht geäußert und erklärt:“... bedurfte es vor der zweiten Impfung keiner erneuter Aufklärung ...."

\section{Unterschrift?}

Im Sachverhalt wird erwähnt, dass die Mutter das Merkblatt nicht unterschrieben hat. Diese fehlende Dokumentation der Zustimmung wurde vom Gericht nicht kritisiert und war im Prozess nicht streitig. Das Gericht sagt jedoch ausdrücklich: "Die Art und Weise der Aufklärung bei der ersten Impfung ... war unter den hier gegebenen Umständen nicht zu beanstanden.” Eine Unterschrift ist also nicht notwendig.

\section{Schlussbemerkung}

Nach wie vor stellt die Aufklärung vor Schutzimpfungen Ärzte vor Probleme. Bemüht um Lösungsvorschläge, hatten wir deshalb ursprünglich beabsichtigt, zusammen mit einigen Kollegen aus der Pädiatrie Leitsätze zur Aufklärung vor Schutzimpfungen zu formulieren. Diese sollten in einer juristischen Fachzeitschrift veröffentlicht werden, weil es nach unserer Auffassung weiterer klärender Hinweise aus der Rechtswissenschaft und der Rechtsprechung bedurfte. Zu groß war bisher die Verunsicherung in der Ärzteschaft. Dieses Vorhaben ist inzwischen durch das bemerkenswerte Urteil des BGH vom 15. Februar 2000 überholt worden.

Unsere Veröffentlichung trägt den Untertitel “Diskussionsbeitrag”. Wir wollen durch diesen Beitrag unsere Kolleginnen und Kollegen dazu anregen, wichtige Urteile fundiert zu analysieren und breit zu diskutieren. Kurz gefasste Meldungen in den Medien führen allzu leicht zu Fehlinterpretationen, Ängsten und Vorurteilen, die sich durch das Studium des Originalurteils beseitigen lassen. Eine interdisziplinäre Diskussion ist nur dann möglich, wenn sich sowohl Juristen als auch Ärzte um eine allgemein verständliche Sprache bemühen und damit von Anfang an Missverständnisse vermeiden. Nur so kann ein Dialog gleichberechtigter Partner zustande kommen und zu befriedigenden Ergebnissen führen.

\section{Fazit - Das Wesentliche in Kürze}

D Die aktuellen Empfehlungen der STIKO sind medizinischer Standard.

D Die empfohlenen Schutzimpfungen im Säuglings- und Kleinkindalter sind Routinemaßnahmen, den Eltern ist der Entscheidungskonflikt durch die öffentlichen Empfehlungen weitgehend abgenommen.

D Den Eltern muss üblicherweise keine Bedenkzeit eingeräumt werden.

D Die Impfung hat deshalb auch nicht an einem gesonderten, von der Aufklärung zeitlich getrennten Termin stattzufinden.

D Es muss über alle spezifischen Risiken der Impfung aufgeklärt werden. 
Dabei kommt es nicht darauf an, ob die möglichen Risiken der Impfung häufig oder selten auftreten.

D Zu Nebenwirkungen und Komplikationen genügt eine Aufklärung im Großen und Ganzen. Die Erläuterung einzelner medizinischer Diagnosen ist nicht erforderlich.

D Zur Aufklärung gehört auch die Beschreibung der impfpräventablen Erkrankung. Auf unnötige Dramatisierung soll verzichtet werden.

D Merkblätter zur Aufklärung sind üblich und haben für den Arzt den Vorteil der späteren Beweisbarkeit.

D Die alleinige Aufklärung durch ein Merkblatt ist nicht ausreichend. Es muss immer Gelegenheit zu einem Gespräch angeboten werden.

D Die Einwilligung zur Impfung kann mündlich erfolgen; eine Unterschrift ist nicht notwendig.

D Bei Routinemaßnahmen wie einer Impfung genügt die Einwilligung eines Elternteiles. Der Arzt kann in der Regel darauf vertrauen, dass der andere Elternteil ebenfalls zustimmt.

D Bei der zweiten Impfung mit dem gleichen Impfstoff im Rahmen einer Grundimmunisierung ist keine erneute Aufklärung erforderlich.

\section{In der Diskussion}

\section{Literatur}

1. BGH NJW 94, 3012-3014

2. Mäulen B (1999) Ärzte unter Anklage - Jeder kann betroffen sein. Dt.Ärztebl.96:A-3091

3. Kramp K (1999) Mehr Rechtssicherheit beim Impfen. Kinder- und Jugendarzt 30:482

4. Kreussler H (1997) Aufklärungspflicht von zunehmender Bedeutung. Dt Ärztebl 94:A-432

5. Endres A (1998) Spektakuläre Prozesse sind die Ausnahme.Dt Ärztebl 95:C-1647-48

6. Reuse S (1999) Jährlich 30000 Klagen wegen ärztlicher Kunstfehler."Die Welt",7.12.

7. Brinkbäumer K, Emcke C, Ludwig U (2000) Tödlicher Pfusch." Der Spiegel" 5/2000

8. Hansis M, Hansis D (1999) Der ärztliche Behandlungsfehler. ecomed, Landsberg:14-19

9. Kautek L (1999) Kinderärzte haften bei Impfschäden. Kinder- und Jugendarzt 30:270

10. Nassauer A, Maass G (1998) Aufklärung vor Schutzimpfungen - Empfehlungen für die Praxis. InfFo I/98:1-7

11. Schlungbaum W (1998) Arzt im Strafrecht. Berliner Ärzte 6/98:25-26

12. Dreher E, Tröndle H (1995) Strafgesetzbuch (Kurzkommentar). Beck'sche Verlagsbuchhandlung, München:1108-1115

13. Berufsverband der Kinder- und Jugendärzte Statement für die Pressekonferenz am 30.8.1999 in Berlin:4-5

14. Brandt K (1997) Frauenarzt muss für Fehler zahlen. Frankfurter Rundschau vom 9.5.

15. Günter R (1997) Indikation für DPT-Schutzimpfung. Entsch. des OLG Koblenz vom 20.1.1995. T\&E Pädiatrie 10:654-656

16. Günter R (1997) Indikation für DPT-Schutzimpfung. Wann verneinte die Rechtsprechung die Indikation. T\&E Pädiatrie 10:728

17. Schneider A, Bierling $G$ (1999) Hygiene und Recht (Entscheidungssammlung). mhp-Verlag, Wiesbaden. Loseblattsammlung, 10/99

18. LG Waldshut-Tiengen (1998) Urteil vom 27.11.1997. Pharma Recht:256-260
19. OLG Stuttgart (2000) Urteil vom 29.12.1998. MedR:35-37

20. Richter H-J (2000) Ein Sumpf von Filz und Amigostrukturen. Der Kassenarzt 3/4:17-18

21. BGH Urteil vom 15.2.2000, VI ZR 48/99, NJW 2000;1784-1788

22. Gerlach FM, Beyer J, Szeczenyi J, Fischer GC (1998) Leitlinien in Klinik und Praxis. Dt Ärztebl 95:A-1014-1021

23. Maass G, Quast U (1987) J Biol Standard 15:185-191

24. Stratton K et al. (1994) Inst of Medicine, National Academy Press, Washington:195

25. Kinnunen $E$ et al. (1998) Am J Epidemiol 147:69-73

26. Stiko-Empfehlungen (1998) Bundesgesundheitsbl:312 